\title{
Crystallization of Curcumin and Cinnamic Acid from Aqueous Solutions of Hydrotropes
}

\author{
Noopur Rathi ${ }^{1}$, Vilas G. Gaikar ${ }^{2 *}$ \\ ${ }^{1}$ Department of Chemical Engineering, Institute of Chemical Technology, Mumbai, India \\ ${ }^{2}$ Dr. Babasaheb Ambedkar Technological University, Maharashtra, India \\ Email: *vg.gaikar@ictmumbai.edu.in
}

How to cite this paper: Rathi, N. and Gaikar, V.G. (2018) Crystallization of Curcumin and Cinnamic Acid from Aqueous Solutions of Hydrotropes. Journal of Crystallization Process and Technology, $\mathbf{8}$ 73-87.

https://doi.org/10.4236/jcpt.2018.83005

Received: July 23, 2018

Accepted: July 28, 2018

Published: July 31, 2018

Copyright $\odot 2018$ by authors and Scientific Research Publishing Inc. This work is licensed under the Creative Commons Attribution International License (CC BY 4.0).

http://creativecommons.org/licenses/by/4.0/

\begin{abstract}
Batch crystallization studies of curcumin from hydrotropic solutions of sodium cumenesulphonate ( $\mathrm{NaCS}$ ) and of cinnamic acid from a photosensitive hydrotropic medium of sodium cinnamate (Na-CIN) were carried out, in an agitated reactor for the effect of alternate heating and cooling cycles on crystal morphology. The crystal characterization by Scanning electron microscopy (SEM) and crystal size distribution (CSD) showed formation of spheroidal curcumin crystals while cinnamic acid formed porous aggregates when subjected to thermal cycles. The UV irradiation of cinnamic acid however showed no formation of the aggregates. The type of hydrotrope used and the initial crystal morphologies of curcumin and cinnamic acid are shown to be important factors to result in a different behaviour of the crystal morphology upon thermal cycles. The CSD data were effectively used for estimation of nucleation and growth rate parameters.
\end{abstract}

\section{Keywords}

Curcumin, Sodium Cinnamate, Sodium Cumenesulphonate, Crystallization, Photosensitive Hydrotrope

\section{Introduction}

Crystallization is usually the final stage of purification of a product as it provides the product of more acceptable purity. Stable crystals with purity, porosity, good flowing properties, better transport and packaging and non-sticky nature are important characteristics required for current industrial applications. A majority of pharmaceutical products are crystallized from volatile organic solvents, such as methanol, acetone, ethyl acetate and dichloromethane, because of ease of their removal by vaporization but many of these solvents are being phased out to 
bring in "green solvents" which are more benign and acceptable. Water can be the most preferred solvent, but suffers from poor solubilisation of many of the drugs. Although, at higher temperatures the drug solubility can be increased, the conditions may not be conducive for applications or the active molecule may degrade because of excessive thermal shock.

We investigate here crystallization from aqueous solutions of hydrotropes which are highly water-soluble amphiphilic salts of sulfonic or carboxylic acids which have been extensively investigated by our group in extraction, solubilization and crystallization processes [1] [2] [3]. Hydrotropes exhibit a remarkable ability of dissolving considerable amounts of sparingly water soluble or otherwise insoluble organic compounds. The solubilization capacity of the hydrotrope solutions is a strong function; usually exponential, of the hydrotrope concentration and mere dilution with water is enough to recover the dissolved materials. Alkyl-aryl carboxylate and sulfonate salts of alkali metals have been widely used as hydrotropes for drug solubilization, detergent formulations and other applications [4]. Solubility enhancement of drugs like Norfloxacin, Telmisartan and Lurasidone hydrochloride etc., which have poor aqueous solubility have been well studied using hydrotropes and mixed hyrdotropy [5] [6] [7]. The efficacy and better separation ability of hydrotrope solutions has been demonstrated for partitioning of phenols between organic solvents and hydrotropic solutions, crystallization of o/p-nitrochlorobenzenes, separation of naphthols, chlorobenzoic acids and extraction of curcuminoids from turmeric [8]-[15]. These studies indicate potential of hydrotropes in separation processes, as an alternative to organic solvents, particularly in pharmaceuticals industry. The recovery of dissolved solute from aqueous hydrotrope solutions is an established fact, yet quantitative analysis of the process is reflected only in few reports.

Reactive precipitation of salicylic acid from sodium salicylate solution was the first report using this novel technique, where sodium salicylate itself as a hydrotrope was shown to dissolve a significant amount of salicylic acid that is formed in the reaction giving an impression of its large supersaturation. [16] The hydrotropic crystallization by dilution with water and by exploiting temperature effect on solubility has been reported for crystallization of curcuminoids, the potential antioxidants from turmeric and that of piperine, an alkaloid responsible for pungency of pepper, both from aqueous solutions of sodium cumenesulphonate [17] [18].

Two different chemical compounds namely, Curcumin (a polyphenolic compound) and an acid which is cinnamic acid were chosen as the model compounds for crystallization study using hydrotropes. Curcumin, being an anti-cancer, anti-inflammatory and sparingly water soluble drug, has many other pharmaceuticals applications [19] [20]. It is also a basic ingredient in food additives and has been studied extensively for it solubility enhancement techniques by formation of solid dispersions, nano-crystals, co-crystals, polymorphs etc. [21] [22] [23] [24]. Cinnamic acid and its derivatives have also shown pharmacologi- 
cal properties including hepatoprotective, anti-oxidant, and anti-diabetic activities [25] [26] [27]. It is also used as an additive and as flavour in food industry.

The use of aqueous solutions, instead of conventional organic solvents is attractive in many food and pharmaceutical industries, where contamination by residual organic solvent is unacceptable. The hydrotropic solubilization of cinnamic acid, aspartic acid, curcumin, thymol, and benzocaine as a function of temperature, using sodium cinnamate (Na-CIN) as photosensitive hydrotrope, has been reported which showed that the trans-cis isomerisation of $\mathrm{Na}-\mathrm{CIN}$ under UV radiation resulted in precipitation of dissolved solutes from the hydrotropic solutions [28]. The use of photosensitive hydrotrope in the crystallization process seems to be an interesting field to be explored, as isomerisation of the photosensitive hydrotrope may result in precipitation of crystals, probably with different morphology, surface characteristics, crystal size and distributions. Recently, classical molecular dynamic simulations studies have shown the effect of hydrotrope on the solubility of the sparingly soluble drugs [29].

The purpose of this paper was to investigate crystal habit of curcumin and cinnamic acid precipitated out of the hydrotropic solutions. Curcumin is crystallized from aqueous sodium cumenesulphonate $(\mathrm{NaCS})$ solutions while cinnamic acid is crystallized from a photosensitive hydrotropic solution of sodium cinnamate (Na-CIN). A unique method of cycles of heating and cooling was adopted to change the crystal shape in the case of curcumin, while for cinnamic acid; the effect of UV radiation is explored.

\section{Materials and Methods}

Cinnamic acid (AR grade) was procured from Molychem India Ltd., Mumbai. Curcumin (95\% pure) was obtained from K. Patel Phyto Extractions Pvt. Ltd. Methanol, NaCS was obtained from M/s. Navdeep Chemicals, Mumbai, and was used as such. Na-CIN was prepared by neutralizing cinnamic acid with sodium hydroxide in methanol in stoichiometric quantities and the precipitated salt thus obtained was thoroughly washed several times with de-ionised water to remove traces of methanol and dried before use.

\subsection{Solubility Studies in Hydrotropic Solutions}

A fully baffled cylindrical glass reactor equipped with a reflux condenser and a stirrer was used in a constant temperature bath. The aqueous hydrotrope solution was maintained at a desired temperature for every run in a range from $30^{\circ} \mathrm{C}$ to $100^{\circ} \mathrm{C}$ for about $6 \mathrm{~h}$ to reach the thermal equilibrium with the bath. The powdered curcumin was added in excess to the solution with constant stirring under reflux conditions. The stirring was stopped at predetermined intervals of time for few minutes to allow the particles to settle down, while the temperature was maintained constant. A small amount of the supernatant solution was then taken in a heated pipette with a filter attached so as to obtain 
clear solution free from any un-dissolved particles and was diluted immediately with enough quantity of distilled water so that the excess curcumin did not precipitate out of the solution. This diluted solution was then checked for its curcumin content using a double beam UV- $V$ is spectrophotometer (Spectrascan UV 2700, Chemito) for the wavelength scan from $200 \mathrm{~nm}$ to $800 \mathrm{~nm}$. The solubility of cinnamic acid in aqueous solutions of Na-CIN was also measured in the same manner.

\subsection{Crystallization Studies of Curcumin and Cinnamic Acid to Modify the Crystal Structure}

A fully baffled jacketed vessel equipped with a reflux condenser, stirrer and heating and cooling arrangement using thermostat (Julabo FP 50) system was used. According to known solubility of curcumin in $2 \mathrm{~mol} \cdot \mathrm{dm}^{-3} \mathrm{NaCS}$ solution, a known weight of curcumin was added to saturate the solution at given temperature. The suspension was first heated to a desired temperature to dissolve all curcumin and the alternate heating and cooling cycles were given to the reactor. The heating was done for $6 \mathrm{~h}$ at $80^{\circ} \mathrm{C}$ and $500 \mathrm{rpm}$ followed by cooling at a constant rate of $0.2^{\circ} \mathrm{C} \cdot \mathrm{min}^{-1}$. Once the reactor temperature reached at $20^{\circ} \mathrm{C}$, it was maintained for $12 \mathrm{~h}$ at the same temperature keeping the stirrer speed at 120 . This procedure was repeated 5 times and after every cooling cycle, the solid crystals were collected and separated from the mother liquor and repeatedly washed with distilled water to remove traces of $\mathrm{NaCS}$, if any. The crystals were then completely dried in an oven at about $100^{\circ} \mathrm{C}$ for $24 \mathrm{~h}$ and checked for Crystal Size Distribution (CSD) and morphology using Beckman Coulter Counter (LS-230) and the Scanning Electron Microscope (SEM), (JEOL, JSM-638OLA, Japan), respectively.

The crystallization of cinnamic acid from aqueous solutions of $\mathrm{Na}$-CIN was carried out in the reactor in a similar manner as mentioned above for curcumin. Crystallization studies were performed in a UV chamber by subjecting the solution to simultaneous exposure to UV radiation using a with Philips $8 \mathrm{~W}$ UV lamp.

The kinetic studies for both curcumin and cinnamic acid were carried out in $\mathrm{NaCS}$ and $\mathrm{Na}-\mathrm{CIN}$ solutions, respectively. The solution was heated to temperature of $80^{\circ} \mathrm{C}$ and maintained for about an hour followed by cooling at a rate of $0.2^{\circ} \mathrm{C} \cdot \mathrm{min}^{-1}$ till the temperature reached $30^{\circ} \mathrm{C}$. The samples were taken after every $30 \mathrm{~min}$ and were analysed for CSD.

\section{Results and Discussion}

\subsection{Solubility Data Interpretations}

Figure 1 shows solubility of curcumin in $2 \mathrm{~mol} \cdot \mathrm{dm}^{-3}$ aqueous $\mathrm{NaCS}$ solution, as a function of temperature. Temperature plays a major role in the absorption spectrum of curcumin and it shows dispersion in water at high temperatures and aggregates upon cooling [30] [31]. With increase in temperature, solubility of 


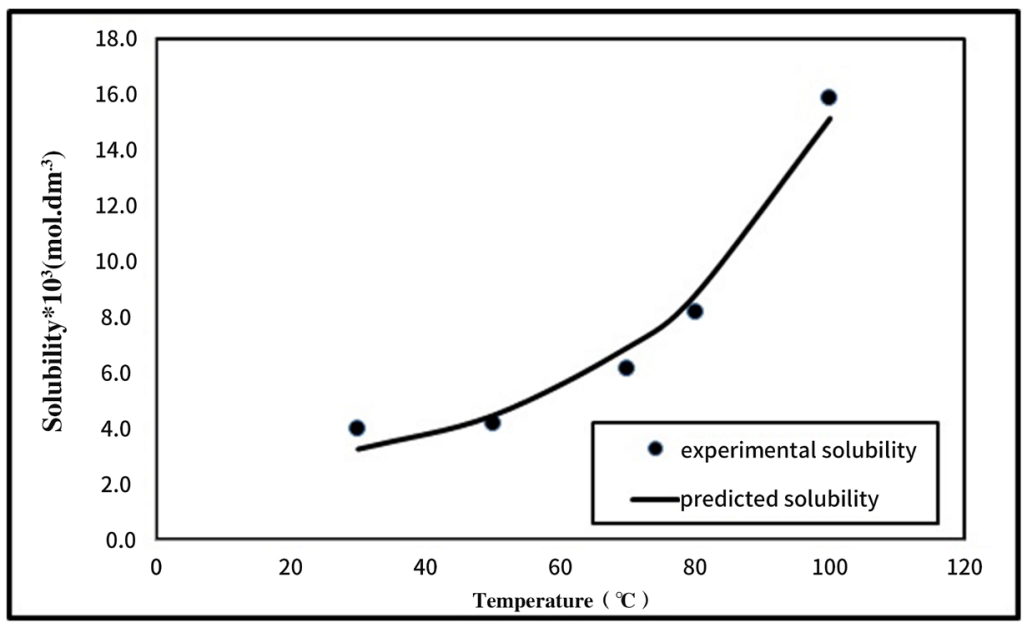

Figure 1. Solubility of curcumin with respect to temperature in $2 \mathrm{~mol} \cdot \mathrm{dm}^{-3} \mathrm{NaCS}$.

curcumin increased exponentially. At $100^{\circ} \mathrm{C}$, the solubility of curcumin was $0.016 \mathrm{~mol} \cdot \mathrm{dm}^{-3}$, which was four times higher than its solubility at $30^{\circ} \mathrm{C}$. The reported solubility of curcuminoids in $2.0 \mathrm{~mol} \cdot \mathrm{dm}^{-3}$ aqueous $\mathrm{NaCS}$ solutions at $\mathrm{pH}$ 3 is $0.008 \mathrm{~mol} \cdot \mathrm{dm}^{-3}$ [11]. Similar solubility values of curcuminoids in aqueous solutions of different hydrotropes such as sodium salicylate, resorcinol and sodium benzoate and a surfactant, sodium lauryl sulphate (SLS) also have been reported [32] [33]. Our other recent studies showed the effect of temperature on solubility of curcumin as well as that of cinnamic acid in aqueous Na-CIN solutions. The maximum solubility of curcumin and cinnamic acid in $1 \mathrm{~mol} \cdot \mathrm{dm}^{-3}$ of $\mathrm{Na}$-CIN are reported to be 0.09 and $0.06 \mathrm{~mol} \cdot \mathrm{dm}^{-3}$ respectively [28].

The increase in solubility of an organic molecule is linked to the aggregation of hydrotropes in aqueous solutions, owing to their amphiphilic nature, in an analogues manner to micelle formation of surfactants [34] [35] [36] [37]. The aggregation of hydrotrope molecules is favoured by its own hydrophobic structure while opposed by electrostatic repulsion between similarly charged head groups; therefore giving an optimum aggregation number $(m)$ of these self assemblies. The total concentration of the hydrotrope $\left(C_{s}\right)$ and monomer concentration $\left[H_{1}\right]$ have been correlated by the following Equation (1) [38] [39] [40]

$$
C_{S}=\frac{2 H_{1}}{m}\left[\left(\frac{m}{2}-1\right)+\exp \left(m K_{2} H_{1}\right)\right]
$$

A solute molecule, when resides in the hydrotrope aggregate; may reduce the electrostatic repulsion between similarly charged head-groups of adjacent hydrotrope molecules in the aggregate. The total amount of the solute associated with the hydrotrope aggregates is given by Equation (2) [39]

$$
S_{T}=2\left(\frac{K_{s}}{K_{2}}\right) \frac{\left[S_{1}\right]}{m^{2}}\left[\exp \left(m K_{2}\left[H_{1}\right]\right)-\left(1+K_{2}\left[H_{1}\right]\right)\right]
$$

where, $S_{1}$ represents concentration of free solute molecules in aqueous phase, i.e. 
water solubility of the solute.The constants, $K_{s}$ and $K_{2}$, represent solute-hydrotrope and hydrotrope-hydrotrope interactions, respectively in Equation (1) and Equation (2) and can be estimated from experimental solubility data as a function of hydrotrope concentration. The amount of a solute associated with the hydrotrope is related to hydrotrope concentration and both are measurable quantities.

The solubility of curcumin in NaCS solution was predicted using the above equations by varying the constants $K_{s}, K_{2}$ and $m$ such that it fits and matches the actual experimental solubility values (see Figure 1 and supplementary information) The optimum values of $K_{s}, K_{2}$ and $m$ are thus obtained which are then compared with the reported values in Table 1 . The $K_{2}$ is much smaller than $K_{s}$ implying weaker hydrotrope-hydrotrope interaction as compared to that between solute and the hydrotrope aggregate. With the increase in temperature, $K_{s}$ increase while $K_{2}$ shows a little variation. Temperature, therefore, can induce a change in the hydrotrope aggregate structures in the solutions, causing more solute molecules to get solubilised in the aggregates. The reported interaction parameters for cinnamic acid in aqueous solutions of $\mathrm{Na}-\mathrm{CIN}$ in reference [28] are also given in Table 1 for comparison.

The SEM images of curcumin crystals after the first and the last cooling cycles are shown in Figure 2(a) and Figure 2(b). The number of fine crystals reduced with the heating and cooling cycles and the crystal surface became much smoother and the shape of the crystals became more spherical. The irregularities on the surface also reduced. The smaller particles which are in the solution and

Table 1. Interaction parameters obtained from modified association model for curcumin crystallized out from $2 \mathrm{~mol} \cdot \mathrm{dm}^{3} \mathrm{NaCS}$ solution.

\begin{tabular}{|c|c|c|c|c|}
\hline & Temperature $\left({ }^{\circ} \mathrm{C}\right)$ & $\begin{array}{c}\mathrm{K}_{\mathrm{s}} \\
\left(\mathrm{dm}^{3} \cdot \mathrm{mol}^{-1}\right)\end{array}$ & $\begin{array}{c}\mathrm{K}_{2} \\
\left(\mathrm{dm}^{3} \cdot \mathrm{mol}^{-1}\right)\end{array}$ & $\begin{array}{l}\text { Aggregation } \\
\text { number }(\mathrm{m})\end{array}$ \\
\hline \multirow{5}{*}{$\begin{array}{l}\text { Curcumin in } \\
\mathrm{NaCS} \text { solution }\end{array}$} & 30 & $181 \pm 2$ & $0.130 \pm 0.002$ & \multirow{5}{*}{22} \\
\hline & 50 & $270 \pm 2$ & $0.137 \pm 0.001$ & \\
\hline & 70 & $310 \pm 3$ & $0.141 \pm 0.002$ & \\
\hline & 80 & $380 \pm 3$ & $0.147 \pm 0.003$ & \\
\hline & 100 & $580 \pm 2$ & $0.148 \pm 0.004$ & \\
\hline \multirow{5}{*}{$\begin{array}{l}\text { Curcumin in } \\
\mathrm{Na}^{-\mathrm{CIN}^{*}}\end{array}$} & 30 & $74 \pm 2$ & $0.070 \pm 0.002$ & \multirow{5}{*}{23} \\
\hline & 40 & $105 \pm 3$ & $0.071 \pm 0.002$ & \\
\hline & 50 & $170 \pm 3$ & $0.072 \pm 0.002$ & \\
\hline & 60 & $198 \pm 4$ & $0.071 \pm 0.002$ & \\
\hline & 90 & $231 \pm 4$ & $0.067 \pm 0.002$ & \\
\hline \multirow{4}{*}{$\begin{array}{l}\text { Cinnamic acid in } \\
\mathrm{Na}^{-\mathrm{CIN}^{*}}\end{array}$} & 30 & $67 \pm 2$ & $0.078 \pm 0.003$ & \multirow{4}{*}{23} \\
\hline & 40 & $96 \pm 3$ & $0.075 \pm 0.002$ & \\
\hline & 60 & $165 \pm 3$ & $0.072 \pm 0.002$ & \\
\hline & 90 & $177 \pm 3$ & $0.073 \pm 0.003$ & \\
\hline
\end{tabular}

*Values taken from the reference [28]. 


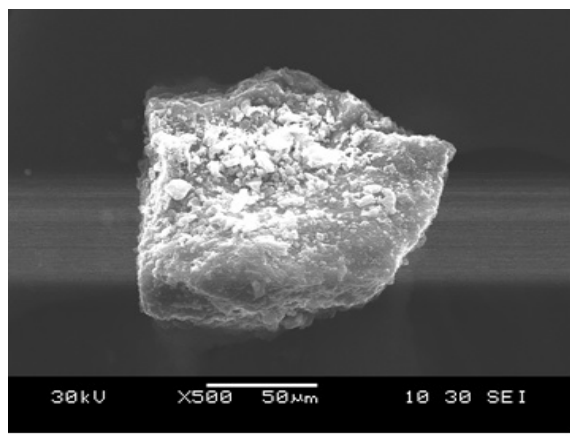

(a)

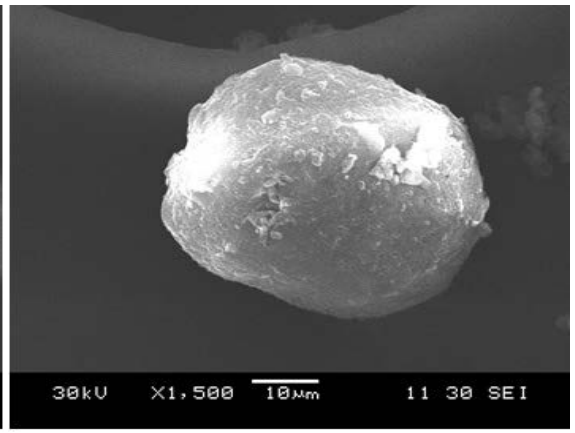

(b)

Figure 2. (a) SEM image of curcumin after $1^{\text {st }}$ cooling cycle; (b) SEM image of curcumin after the $5^{\text {th }}$ cooling cycle.

adhering to the surface of the larger crystals tend to dissolve fast while larger crystals have a lower rate of dissolution due to the phenomenon of Ostwald's ripening.

With the increase in temperature, the irregularities and the smaller particles tend to dissolve first. When the temperature is reduced, the precipitation occurs on the surface of the larger particles as they serve as the nucleation sites for the new particle to grow. According to the Gibbs-Volmer theory, the units of crystallizing substance arriving at the crystal face do not immediately form intocrystal lattice and the equilibrium is established between the growing layer and the bulk solution [41]. Thus, alternate heating and cooling resulted in the smaller but smoother and more uniform spheroidal crystals of curcumin.

Figures 3(a)-(c) show SEM images of cinnamic acid, the crystals obtained after the first cooling cycle and those after the $5^{\text {th }}$ cooling cycle, respectively. The study was done to investigate crystal morphology changes with increasing temperature differences between the heating and cooling temperatures. The crystals of pure cinnamic acid are irregular but are flat plate type flakes, with smooth surfaces. The crystals after the $1^{\text {st }}$ cooling cycle are smaller sharp needles. Although there is no apparent increase in individual crystal size, but they form agglomerates of uniform size after repeated cooling cycles. These agglomerates are porous in nature with non-uniform surface. After the repeated heating and cooling cycles, the amount of the fine particles reduced significantly. The particle size too became uniform. The heating provides the energy to the particles to dissolve. The surface of the crystals or particles is the part which gets solubilized first but growth starts with surface of least energy.

The crystallization of cinnamic acid in the presence of UV radiation showed a difference in the crystal habit from that of crystals obtained without exposure to UV radiation. Figure 3(d) shows that although there is aggregation of the crystals but the aggregates are not compact or properly clustered and there are more fine particles present in the samples. Similar crystal structures for cinnamic acid, before and after UV radiations, can be seen in the earlier literature [28]. The UV irradiation is responsible conversion of trans-Na-CIN form to the cis-Na-CIN 


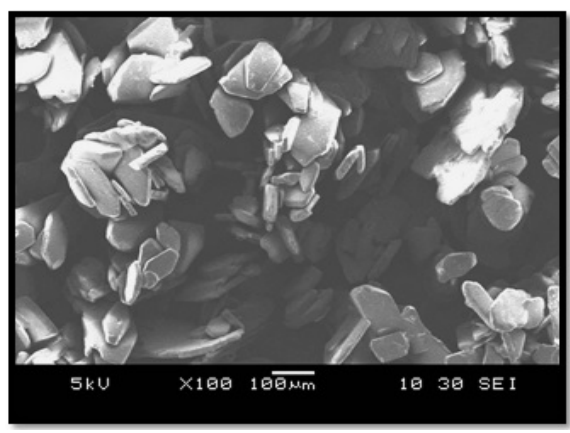

(a)

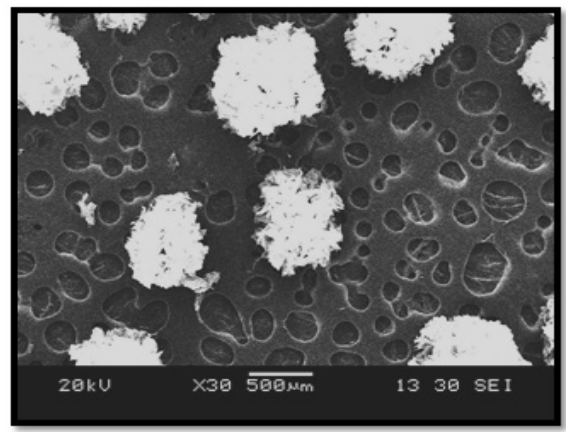

(c)

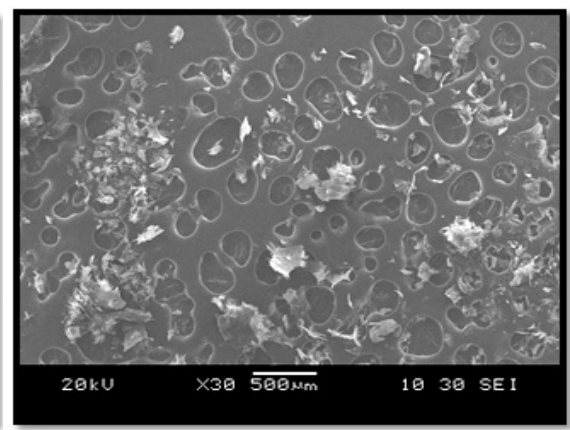

(b)

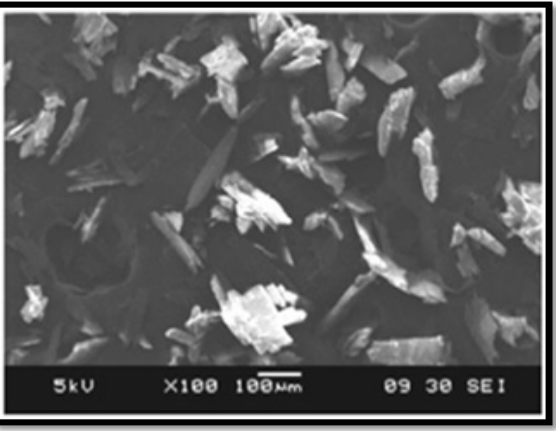

(d)

Figure 3. SEM images of (a) Pure Cinnamic acid; (b) Cinnamic acid after $1^{\text {st }}$ cooling cycle; (c) Cinnamic acid after $5^{\text {th }}$ cooling cycle; (d) After the UV radiation.

form. The cinnamic acid, itself being a photosensitive molecule, also converts to its cis formon exposure to the UV light. It is quite obvious that presence of another form of a molecule interferes in the crystal building process. The clusters are not well defined and the crystals remain more as individuals rather than forming agglomerates. The shape of the crystals is also not uniform and they tend to form thin, almost transparent flake like crystals.

Thus it could be observed that alternate thermal cycles did not result into similar morphologies for both curcumin and cinnamic acid. The type of hydrotrope used and the initial morphologies of curcumin and cinnamic acid are also crucial for the final morphology of the crystals obtained.

\subsection{CSD Data Interpretation and Kinetics of Crystallization}

The CSD is very useful and effective tool for obtaining the kinetics of crystallization reactions. A crystal population density or the number density (n), can be defined as number of crystals per unit size per unit volume of the system and is defined by Equation (3) [42]

$$
\lim _{\Delta L \rightarrow 0} \frac{\Delta N}{\Delta L}=\frac{\mathrm{d} N}{\mathrm{~d} L}=n
$$

where, $\Delta N$ is number of crystals in the size range $\Delta L$ per unit volume. The value of $n$ depends on the value of $L$ at which the interval $\mathrm{d} L$ is taken. The number of crystals in the size range $L_{1}$ to $L_{2}$ is thus given by Equation (4). 


$$
\Delta N=\int_{L 1}^{L 2} n \mathrm{~d} L
$$

From the CSD data, the crystals of curcumin which had an average size of 83 $\mu \mathrm{m}$ reduced to 66.3 and $30.7 \mu \mathrm{m}$ after the $2^{\text {nd }}$ and the $5^{\text {th }}$ alternate cycles. Figure 4(a) and Figure 4(b) show the CSD for curcumin crystals after $2^{\text {nd }}$ and $5^{\text {th }}$ cycles. The CSD became narrower, more uniform and the number of fine crystals reduced.

For cinnamic acid, the four different regions of size distributions reduced to merely two or three regions. Figures 4 (c)-(e) show the CSD of pure cinnamic acid, cinnamic acid after the $1^{\text {st }}$ and the $5^{\text {th }}$ cooling cycles, respectively. Pure cinnamic acid has a narrow range of particle size with having a mean diameter of $176 \mu \mathrm{m}$.

Table 2 shows the mean diameter of curcumin and cinnamic acid crystals, after every cooling cycle i.e. after every $30 \mathrm{~min}$. CSD is contributed by both nucleation and growth phase of the crystal. The kinetic parameters of the crystallization were estimated from the CSD data assuming that the growth rate is independent of the size. The relationship between the crystal size $(L)$ and the population density $(n)$, which is number of crystals per unit size and per unit volume at steady state conditions is given by Equation (5) [42] [43].

$$
n=n_{0} \exp \left(-\frac{L}{G \tau}\right)
$$

where, $n_{0}$ is the population density of zero sized crystal and $\tau$ is residence time. The nucleation rate $B$ and the growth rate $G=\frac{\mathrm{d} L}{\mathrm{~d} t}$ can be related to each other as given in Equation (6).

$$
B=n_{0} G
$$

Figure 5 shows the variation of $L$ with the time for both curcumin and cinnamic acid. For curcumin, it shows a decrease with time while for cinnamic acid

Table 2. Crystallization kinetic parameters for curcumin and cinnamic acid.

\begin{tabular}{ccccccc}
\hline Kinetics & Time $(\min )$ & $\operatorname{Avg~L}(\mu \mathrm{m})$ & $\log \mathrm{n}$ & $\mathrm{n}_{\mathrm{o}}\left(\mathrm{m}^{-4}\right)$ & $\mathrm{G}\left(\mathrm{m} \cdot \mathrm{s}^{-1}\right)$ & $\mathrm{B}\left(\mathrm{m}^{-3} \cdot \mathrm{s}^{-1}\right)$ \\
\hline & 30 & $80 \pm 5$ & 13.6 & & & \\
$\begin{array}{c}\text { Curcumin from } \\
\text { aqueous }\end{array}$ & 60 & $66 \pm 8$ & 13.8 & & & \\
$\begin{array}{c}\text { solutions of } \\
\text { NaCS }\end{array}$ & 90 & $44 \pm 6$ & 14.3 & $3.48 \mathrm{E}+15$ & $1.34 \mathrm{E}-06$ & $4.68 \mathrm{E}+09$ \\
& 120 & $36 \pm 9$ & 14.6 & & & \\
\hline & 150 & $30 \pm 5$ & 14.8 & & & \\
$\begin{array}{c}\text { Cinnamic acid } \\
\text { from aqueous }\end{array}$ & 60 & $275 \pm 8$ & 12.0 & & & \\
solutions of & 90 & $302 \pm 6$ & 11.9 & $5.48 \mathrm{E}+13$ & $5.85 \mathrm{E}-06$ & $3.21 \mathrm{E}+08$ \\
Na-CIN & 120 & $318 \pm 5$ & 11.9 & & & \\
& 150 & $326 \pm 5$ & 11.8 & & & \\
\hline
\end{tabular}




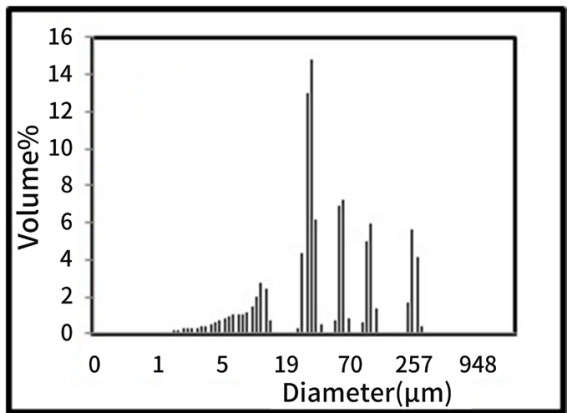

(a)

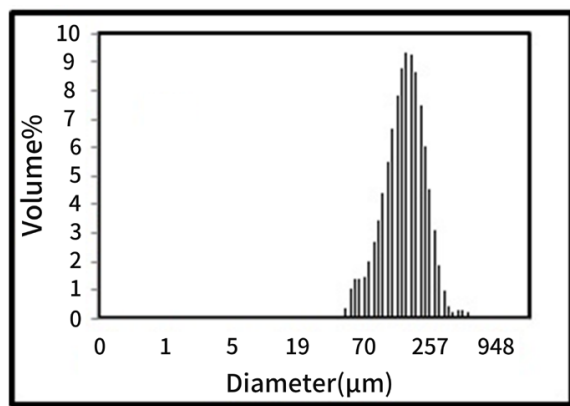

(c)

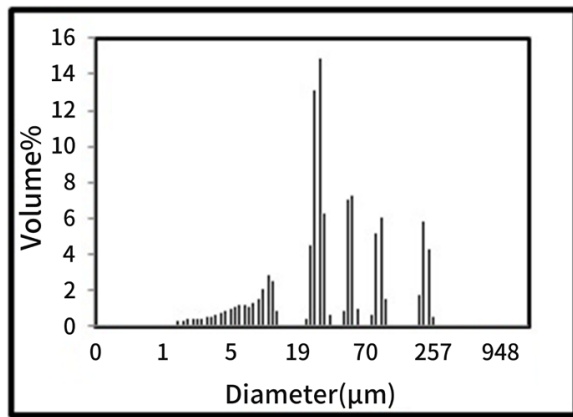

(b)

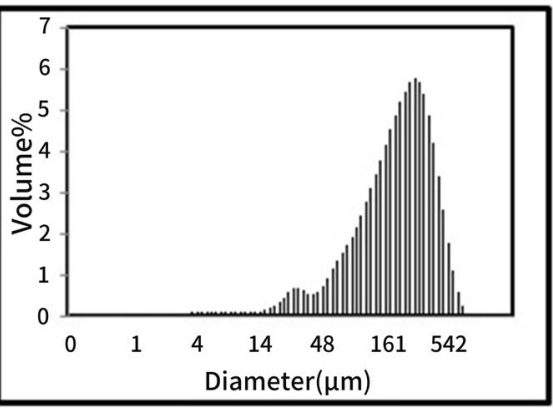

(d)

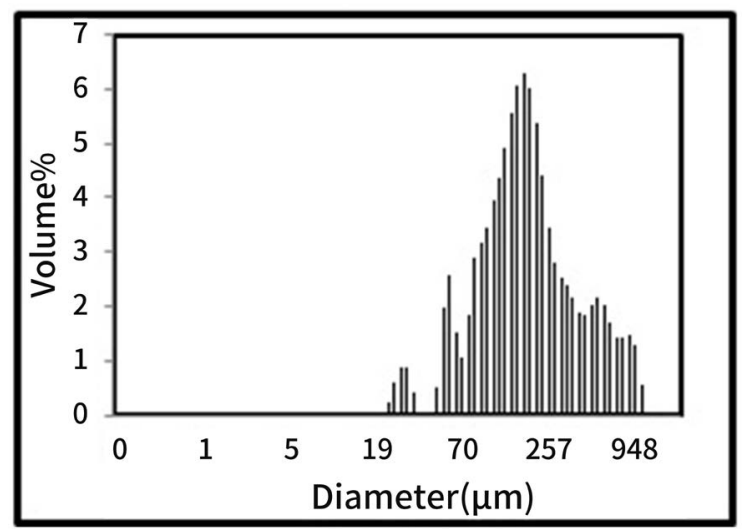

(e)

Figure 4. CSD of curcumin crystallized out from $2 \mathrm{~mol} \cdot \mathrm{dm}^{-3} \mathrm{NaCS}$ solution (a) after $1^{\text {st }}$ cycle; (b) after $5^{\text {th }}$ cycle; and (c) Pure cinnamic acid, CSD of Cinnamic acid crystallized from $0.45 \mathrm{~mol} \cdot \mathrm{dm}^{3}$ of Na-CIN solution; (d) After $1^{\text {st }}$ cooling cycle; (e) After $5^{\text {th }}$ cooling cycle.

it shows that the growth of the crystal increases and limits in the first $100 \mathrm{~min}$, indicating that the surface energy was minimized and the crystal shape has become stable. This difference in size variation with time for curcumin and cinnamic acid can be due to the fact that the cinnamic acid forms aggregates and thus shows overall increase in size, although the individual crystallize for both curcumin and cinnamic acid decreases. The volume percentage $V$ obtained from the CSD graph for the mean value of the crystal size $L$ was equated to the number of crystals as given by Equation (7),

$$
V=v \cdot n
$$




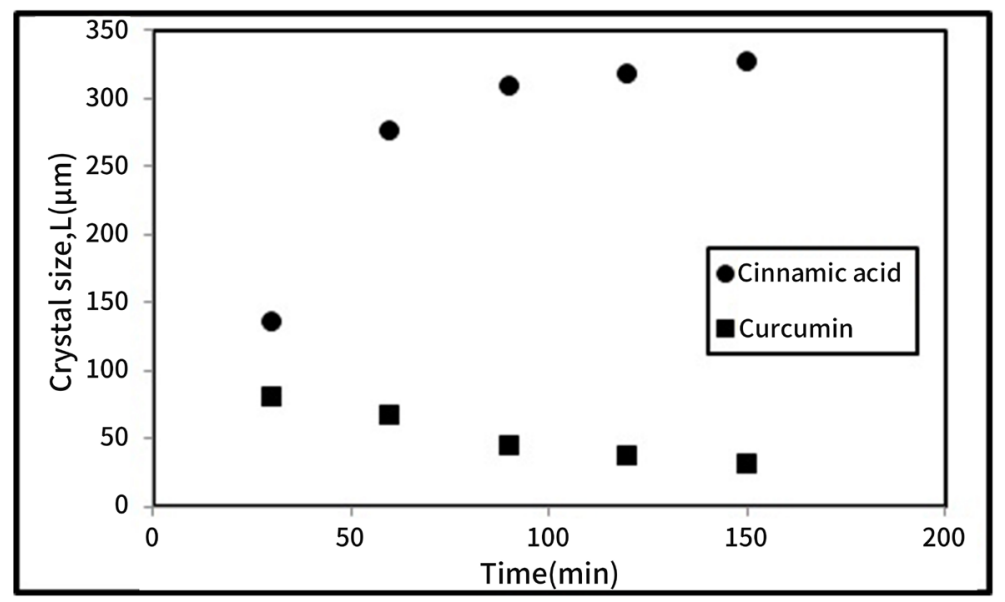

Figure 5. Variation of average crystal size $L(\mathrm{~nm})$ with time for curcumin and cinnamic acid from aqueous solutions of $\mathrm{NaCS}$ and $\mathrm{Na}-\mathrm{CIN}$ respectively.

where, $v$ is the volume of one crystal of the mean size $L$ and $n$ is the population density or the crystal density. The values of $n$ as obtained from Equation (7) are given in Table 2. Thus $\log n$ obtained was plotted against $L$ and the constants $n_{0}$ and the slope $\left(-\frac{L}{G \tau}\right)$ were estimated. The growth rate $\mathrm{G}$ and $\mathrm{B}$ are calculated using equations from (5) to (7) and are reported in Table 2.

These studies have brought out interesting aspects of the crystallization of organic molecules from aqueous solutions. Precipitation from aqueous solutions is definitely attractive because of green label associated with water as solvent. The hydrotropes being highly water soluble, are not expected to precipitate with the solutes while water washing given to the precipitated solids can not only remove the adhering layer of hydrotrope solutions on the solid surface but also the entire amount of wash water can be used for dilution of the solution in the main crystallization step by dilution, if required. Concentrating the diluted hydrotrope solutions to the desired level by evaporation, however, forms the major energy penalty in the process. Therefore, sensible heat transfer for heating and cooling can be more acceptable process. Since the temperature is also limited by boiling point of the aqueous solutions, even thermally sensitive materials can be purified from the aqueous solutions. The most important point, however, is the different crystal morphology, that one can achieve with these solutions.

\section{Conclusion}

Hydrotropes were successfully utilised for crystallization process to obtain crystals of curcumin and cinnamic acid using alternate heating and cooling cycles. Curcumin was crystallized from aqueous $\mathrm{NaCS}$ solutions to obtain more uniform spheroidal crystals of smaller size and smoother surface. The crystallization of cinnamic acid in the presence of photosensitive hydrotrope $\mathrm{Na}-\mathrm{CIN}$, however, resulted in formation of porous aggregate structures which should give a lower 
bulk density solid and probably higher surface area. But, the same crystallization process which was subjected to UV radiation resulted in the different morphology of cinnamic acid. It was seen that the obtained morphologies of curcumin and cinnamic acid depended on the initial crystal morphologies of curcumin and cinnamic acid, the type of hydrotrope used and the UV irradiation. Alternate heating and cooling cycles were proved to be an efficient methodology to obtain unique and different crystal habits of the compounds. The solubility values and kinetics parameters of the crystallization processes are important in designing crystallizers and scale-up.

\section{Acknowledgements}

The author wishes to thank University Grants Commission (UGC), India, for award of Junior Research Fellowship. The authors are also thankful to the Institute of Chemical Technology, Mumbai, India for providing experimental and analytical facilities.

\section{Conflicts of Interest}

The authors declare no conflicts of interest regarding the publication of this paper.

\section{References}

[1] Roy, B.K. and Moulik, S.P. (2002) Functions of Hydrotropes (Sodium Salicylate, Proline, Pyrogallol, Resorcinol and Urea) in Solution with Special Reference to Amphiphile Behaviors. Colloids and Surfaces A: Physicochemical and Engineering Aspects, 203, 155-166. https://doi.org/10.1016/S0927-7757(01)01099-8

[2] Jain, P., Goel, A., Sharma, S. and Parmar, M. (2010) Solubility Enhancement Techniques with Special Emphasis on Hydrotrophy. International Journal Of Pharma Professional s Research, 1, 34-45.

[3] Agarwal, M. and Gaikar, V.G. (1992) Extractive Separations Using Hydrotropes. Separation Science and Technology, 2, 79-84.

[4] Balasubramanian, D., Srinivas, V., Gaikar, V.G. and Sharma, M.M. (1989) Aggregation Behavior of Hydrotropic Compounds in Aqueous Solution. The Journal of Physical Chemistry, 93, 3865-3870. https://doi.org/10.1021/j100346a098

[5] Girishpai, K., Divya, S., Reddy, M.S., Kumar, L. and Vamshi, K.T. (2014) Solubility Enhancement of Norfloxacin by Hydrotropy Technique. International Journal of Pharmacy and Pharmaceutical Sciences, 6, 395-397.

[6] Jyotsana, R.M., Kiran, T.P. and Kamal, D. (2015) Solubility Enhancement Studies on Lurasidone Hydrochloride Using Mixed Hydrotropy. International Journal of Pharmaceutical Investigation, 5, 114-120. https://doi.org/10.4103/2230-973X.153390

[7] Phulzalke, S.B., Kate, B.A. and Bagade, M.Y. (2015) Solubility Enhancement of Telmisartan Using Mixed Hydrotropy Approach. Asian Journal of Biomedical and Pharmaceutical Sciences, 5, 37-39.

[8] Negi, A.S. and Gaikar, V.G. (2009) Partitioning of $o / p$-Nitrophenols in the Presence of Hydrotropes in Aqueous Solutions. Separation Science and Technology, 44, 734-752. https://doi.org/10.1080/01496390802625768 
[9] Raynaud-Lacroze, P.O. and Tavare, N.S. (1993) Separation of 2-Naphthol: Hydrotropy and Precipitation. Industrial \& Engineering Chemistry Research, 32, 685-691. https://doi.org/10.1021/ie00016a015

[10] Colonia, E.J., Dixit, A.B. and Tavare, N.S. (1998) Separation of Chlorobenzoic Acids through Hydrotropy. Industrial \& Engineering Chemistry Research, 37, 1956-1969. https://doi.org/10.1021/ie970686c

[11] Dandekar, D.V. and Gaikar, V.G. (2003) Hydrotropic Extraction of Curcuminoids from Turmeric. Separation Science and Technology, 38, 1185-1215.

https://doi.org/10.1081/SS-120018130

[12] Dandekar, D.V., Jayaprakasha, G.K. and Bhimanagouda, S.P. (2008) Simultaneous Extraction of Bioactive Limonoid Aglycones and Glucoside from Citrus aurantium L. Using Hydrotropy. Food Chemistry, 109, 515-520. https://doi.org/10.1016/j.foodchem.2007.12.071

[13] Ramesh, N., Jayakumar, C. and Gandhi, N.N. (2009) Effective Separation of Petro Products through Hydrotropy. Chemical Engineering \& Technology, 32, 129-133. https://doi.org/10.1002/ceat.200800328

[14] Mohanasundaram, R., Jayakumar, C. and Gandhi, N.N. (2010) Separation of Styrene-Ethyl Benzene Mixture through Hydrotropy. International Journal of Applied Science and Engineering, 8, 1-9.

[15] Subbarao, C.V., Chakravarthy, I.P.K., SaiBharadwaj, A.V.S.L. and Prasad, K.M.M. (2012) Functions of Hydrotropes in Solutions. Chemical Engineering \& Technology, 35, 225-237. https://doi.org/10.1002/ceat.201100484

[16] Tavare, N.S. and Gaikar, V.G. (1991) Precipitation of Salicyclic Acid: Hydrotropy and Reaction. Industrial \& Engineering Chemistry Research, 30, 722-728.

https://doi.org/10.1021/ie00052a015

[17] Dandekar, D.V. and Gaikar, V.G. (2003) Precipitation of Curcuminoids from Hydrotrope Solution: Crystal Nucleation and Growth Kinetics from Batch Experiments. Separation Science and Technology, 38, 3625-3644. https://doi.org/10.1081/SS-120024221

[18] Gaulkar, S.U. and Gaikar, V.G. (2004) Precipitation of Piperine from Hydrotropic Solutions: Study of Crystal Nucleation and Growth Kinetics from Batch Experiments. Separation Science and Technology, 39, 3431-3452. https://doi.org/10.1081/SS-200034344

[19] Ramadasn, K., Bhanumathy, P., Nirmala, K. and George, M.C. (1985) Potential Anticancer Activity of Turmeric (Curcuma longa). Cancer Letters, 29, 197-202. https://doi.org/10.1016/0304-3835(85)90159-4

[20] Revathy, S., Elumalai, S., Benny, M. and Antony, B. (2011) Isolation, Purification and Identification of Curcuminoids from Turmeric (Curcuma longa L.) by Column Chromatography. Journal of Experimental Biology, 2, 21-25.

[21] Teixeira, C.C.C., Mendonça, L.M., Bergamaschi, M.M., Queiroz, R.H.C., Souza, G.E.P., Antunes, L.M.G. and Freitas, L.A.P. (2016) Microparticles Containing Curcumin Solid Dispersion: Stability, Bioavailability and Anti-Inflammatory Activity. AAPS PharmSciTech, 17, 252-261. https://doi.org/10.1208/s12249-015-0337-6

[22] He, Y., Huang, Y. and Cheng, Y. (2010) Structure Evolution of Curcumin Nanoprecipitation from a Micromixer. Crystal Growth \& Design, 10, 1021-1024. https://doi.org/10.1021/cg9009916

[23] Thakuria, R., Delori, A., Jones, W., Lipert, M.P., Roy, L. and Rodriguez-Horned, N. (2013) Pharmaceutical Cocrystals and Poorly Soluble Drugs. International Journal of Pharmaceutics, 453, 101-125. https://doi.org/10.1016/j.ijpharm.2012.10.043 
[24] Thorat, A.A. and Dalvi, S.V. (2015) Solid-State Phase Transformations and Storage Stability of Curcumin Polymorphs. Crystal Growth \& Design, 15, 1757-1770. https://doi.org/10.1021/cg501814q

[25] Lee, E.J., Kim, S.R., Kim, J. and Kim, Y.C. (2002) Hepatoprotective Phenylpropanoids from Scrophularia buergeriana Roots against $\mathrm{CCl}_{4}$-Induced Toxicity: Action Mechanism and Structure-Activity Relationship. Planta Medica, 68, 407-411. https://doi.org/10.1055/s-2002-32081

[26] Natella, F., Nardini, M., Di Felice, M. and Scaccini, C. (1999) Benzoic and Cinnamic Acid Derivatives as Antioxidants: Structure-Activity Relation. Journal of Agricultural and Food Chemistry, 47, 1453-1459. https://doi.org/10.1021/jf980737w

[27] Liu, I.M., Hsu, F.L., Chen, C.F. and Cheng, J.T. (2000) Antihyperglycemic Action of Isoferulic Acid in Streptozotocin-Induced Diabetic Rats. British Journal of Pharmacology, 129, 631-636. https://doi.org/10.1038/sj.bjp.0703082

[28] Devendra, L.P. and Gaikar, V.G. (2012) Is Sodium Cinnamate a Photos Witch Able Hydrotrope? Journal of Molecular Liquids, 165, 71-77. https://doi.org/10.1016/j.molliq.2011.10.010

[29] Das, S. and Paul, S. (2016) Computer Simulation Studies of the Mechanism of Hydrotrope-Assisted Solubilization of a Sparingly Soluble Drug Molecule. The Journal of Physical Chemistry B, 120, 3540-3550. https://doi.org/10.1021/acs.jpcb.5b11902

[30] Jagannathan, R., Abraham, P.M. and Poddar, P. (2012) Temperature-Dependent Spectroscopic Evidences of Curcumin in Aqueous Medium: A Mechanistic Study of Its Solubility and Stability. The Journal of Physical Chemistry B, 116, 14533-14540. https://doi.org/10.1021/jp3050516

[31] Kurien, B.T., Singh, A., Matsumoto, H. and Scofield, R.H. (2007) Improving the Solubility and Pharmacological Efficacy of Curcumin by Heat Treatment. ASSAY and Drug Development Technologies, 5, 567-576. https://doi.org/10.1089/adt.2007.064

[32] Sreenivasan, D., Jayakumar, C. and Gandhi, N.N. (2010) Effect of Hydrotropes on Solubility and Mass Transfer Co-Efficient of Curcuminoids. Journal of Pharmacy Research, 3, 2170-2171.

[33] Rahman, S.M.H., Telny, T.C., Ravi, T.K. and Kuppusamy, S. (2009) Role of Surfactant and $\mathrm{pH}$ in Dissolution of Curcumin. Indian Journal of Pharmaceutical Sciences, 71, 139-142. https://doi.org/10.4103/0250-474X.54280

[34] Srinivas, V., Rodley, G.A., Ravikumar, K., Robinson, W.T., Turnbull, M.M. andBalasubramanian, D. (1997) Molecular Organization in Hydrotrope Assemblies. Langmuir, 13, 3235-3239. https://doi.org/10.1021/la9609229

[35] Rodriguez-Hornedo, N. and Murphy, D. (2004) Surfactant-Facilitated Crystallization of Dihydrate Carbamazepine during Dissolution of Anhydrous Polymorph. Journal of Pharmaceutical Sciences, 93, 449-460. https://doi.org/10.1002/jps.10496

[36] Kaival, P., Kulkarni, P.K., Dixit, M. and Kni, A.G. (2011) Influence of Surfactants on Crystal Form of Mefenamic Acid. Thai Journal of Pharmaceutical Sciences, 35, 40-50.

[37] Ahmed, M.A., Rhgigh, A.M. and Shakeel, F. (2009) Effect of Surfactants on the Crystal Properties and Dissolution Behavior of Aspirin. Asian Journal of Research in Chemistry, 2, 202-206.

[38] Geetha, K.K., Tavare, N.S. and Gaikar, V.G. (1991) Separation of $o$ and $p$ Chloronitrobenzenes through Hydrotropy. Chemical Engineering Communications, 102, 211-224. https://doi.org/10.1080/00986449108910857 
[39] Raman, G. and Gaikar, V.G. (2002) Extraction of Piperine from Piper Nigrum (Black Pepper) by Hydrotropic Solubilization. Industrial \& Engineering Chemistry Research, 41, 2966-2976. https://doi.org/10.1021/ie0107845

[40] Sharma, R.A. and Gaikar, V.G. (2012) Hydrotropic Extraction of Reserpine from Rauwolfiavomitoria Roots. Separation Science and Technology, 47, 827-833. https://doi.org/10.1080/01496395.2011.635623

[41] Volmer, M. and Weber, A.Z. (1926) Nucleus Formation in Supersaturated Systems. Zeitschrift für Physikalische Chemie, 119, 277-301.

[42] Mullin, J.W. (2001) Crystallization. 4th Edition, Butterworth-Heinemann, Woburn.

[43] Randolph, A.D. and Larson, M.A. (1986) Theory of Particulate Processes. 2nd Edition, Academic Press, New York.

\section{Symbols}

m: optimum aggregation number

$C_{s}$ : total concentration of the hydrotrope $\left(\mathrm{mol} \cdot \mathrm{dm}^{3}\right)$

$\left[H_{1}\right]$ : monomer concentration $\left(\mathrm{mol} \cdot \mathrm{dm}^{3}\right)$

$S_{1}$ : concentration of free solute molecules in aqueous phase $\left(\mathrm{mol} \cdot \mathrm{dm}^{3}\right)$

$K_{s}$ : solute- hydrotrope interactions $\left(\mathrm{dm}^{3} \cdot \mathrm{mol}^{-1}\right)$

$K_{2}$ : hydrotrope- hydrotrope interactions $\left(\mathrm{dm}^{3} \cdot \mathrm{mol}^{-1}\right)$

$n$ : crystal density or population density $\left(\mathrm{m}^{-4}\right)$

$\Delta L$ : crystal size range $(\mathrm{m})$

$\Delta N$ : number of crystals in the size range $\Delta L$

$n_{0}$ : population density of zero sized crystal (no. of particles. $\mathrm{m}^{-3}$ )

$\tau$ : residence time $(\mathrm{s})$

$B$ : nucleation rate $\left(\mathrm{m}^{-3} \cdot \mathrm{min}^{-1}\right)$

$G$ : growth rate $\left(\mathrm{m} \cdot \mathrm{min}^{-1}\right)$

$V$ : totalvolume of the crystals $\left(\mathrm{m}^{3}\right)$

$v$. volume of single crystal of mean size $\mathrm{L}\left(\mathrm{m}^{3}\right)$ 